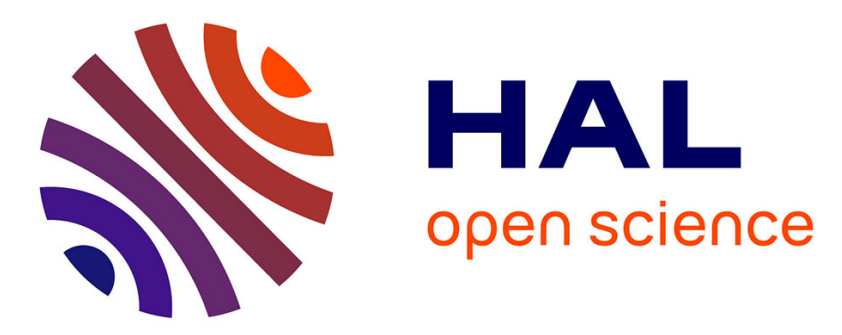

\title{
Détermination des densités électroniques par cavité hyperfréquence et du taux de destruction des atomes métastables du néon
}

J. Muzart, André Ricard, J.-M. Rocard

\section{- To cite this version:}

J. Muzart, André Ricard, J.-M. Rocard. Détermination des densités électroniques par cavité hyperfréquence et du taux de destruction des atomes métastables du néon. Revue de Physique Appliquée, 1971, 6 (2), pp.233-235. 10.1051/rphysap:0197100602023300 . jpa-00243533

HAL Id: jpa-00243533

https://hal.science/jpa-00243533

Submitted on 1 Jan 1971

HAL is a multi-disciplinary open access archive for the deposit and dissemination of scientific research documents, whether they are published or not. The documents may come from teaching and research institutions in France or abroad, or from public or private research centers.
L'archive ouverte pluridisciplinaire HAL, est destinée au dépôt et à la diffusion de documents scientifiques de niveau recherche, publiés ou non, émanant des établissements d'enseignement et de recherche français ou étrangers, des laboratoires publics ou privés. 


\title{
" DÉTERMINATION DES DENSITÉS ÉLECTRONIQUES PAR CAVITÉ HYPERFRÉQUENCE ET DU TAUX DE DESTRUCTION DES ATOMES MÉTASTABLES DU NÉON »
}

\author{
J. MUZART, A. RICARD et J.-M. ROCARD
}

Laboratoire de Physique des Milieux Ionisés, Faculté des Sciences de Toulouse

\begin{abstract}
Résumé. - Des mesures simultanées de $n_{\mathrm{e}}$ (cavité résonnante) et de $\mathrm{Ne}^{*}\left({ }^{3} \mathrm{P}_{0.2}\right)$ (absorption optique) en fonction de la pression et du courant d'une décharge luminescente de Néon ont permis la détermination du taux de destruction des atomes métastables par collisions électroniques : $\left\langle\sigma_{r x} v\right\rangle=1,4 \times 10^{-7} \mathrm{~cm}^{3} \mathrm{~s}^{-1}$.
\end{abstract}

Abstract. - Simultaneous measurements of $n_{\mathrm{e}}$ (cavity method) and $\mathrm{Ne}^{*}\left({ }^{3} \mathrm{P}_{0.2}\right)$ (optical absorption) as a function of $p$ and $I$ (pressure and current in a Neon glow discharge) have led to the determination of the metastable destruction rate by electronic collisions : $\left\langle\sigma_{r x} v\right\rangle=1.4 \times 10^{-7} \mathrm{~cm}^{3} \mathrm{~s}^{-1}$.

Introduction. - Nous avons construit un appareillage qui nous a permis de déterminer simultanément les concentrations des électrons et des atomes métastables du Néon dans la colonne positive d'une décharge de ce gaz et d'en déduire le taux de destruction $\left\langle\sigma_{r x} v\right\rangle$ de ces métastables par collisions électroniques. Pour éviter que ce taux de destruction ne soit principalement dû aux impuretés présentes dans la décharge, certaines précautions ont été prises pour que le système à vide soit aussi propre que possible. Le dispositif expérimental concernant la décharge proprement dite est constitué des éléments suivants : un système à vide étuvable (vide limite $10^{-7}$ torr), le tube à décharge en quartz de $8 \mathrm{~mm}$ de diamètre et de $20 \mathrm{~cm}$ de longueur, une alimentation stabilisée en tension.

Les densités électroniques sont mesurées à l'aide d'une cavité cylindrique résonnant sur le mode $\mathrm{TE}_{011}$ $\left(f_{0}=9125 \mathrm{MHz}\right.$ sans plasma); la fréquence du klystron peut varier linéairement en fonction du temps (grâce à un micromoteur synchrone qui déplace la vis de réglage du klystron) et les déterminations des écarts de fréquence $\Delta f$ de résonance (de la cavité avec et sans plasma) sont effectuées à l'aide d'un enregistreur double voie permettant d'obtenir simultanément les courbes de résonance de la cavité et de l'ondemètre quand la fréquence du klystron varie.

Compte tenu de la théorie des petites perturbations [1], [2], la densité électronique $n_{\mathrm{e}}$ et la fréquence de collision électron-neutre $v_{1}$ sont données par les relations suivantes :

$$
\left(\mathrm{cm}^{-3}\right) n_{\mathrm{e}}=3,6 \times 10^{4} \Delta f \cdot\left(1+\frac{v_{1}^{2}}{\omega^{2}}\right),
$$

et

$$
\left(\mathrm{s}^{-1}\right) v_{1}=\frac{\pi f_{0}^{2} \Delta(1 / Q)}{\Delta f}
$$

où $Q$ est le coefficient de surtension de la cavité. Le dispositif de mesure par absorption optique des atomes métastables a été décrit dans une étude antérieure [3].

Résultats. - 1. Densités ÉLECTRONIQUES. - Compte tenu des relations (1) et (2), nous pouvons déterminer la densité électronique et la fréquence de collision dans la colonne positive d'une décharge luminescente de Néon. Nous avons vérifié que pour des pressions inférieures à 5 torrs, la fréquence de collision était négligeable devant la fréquence de l'onde $\left(v_{1}^{2} \ll \omega^{2}\right)$; par contre, pour $p=10$ torrs, nous avons trouvé expérimentalement les valeurs suivantes :
$I(\mathrm{~mA})$
5
10
20
$v_{1}(\mathrm{rad} / \mathrm{s})$
$1,5 \times 10^{10}$
$1,9 \times 10^{10}$
$2,3 \times 10^{10}$

dans ce cas, nous avons tenu compte du facteur de correction $\left(1+v_{1}^{2} / \omega^{2}\right)$ pour la détermination de la densité électronique.

Nous avons rassemblé sur la figure 1 les résultats concernant les densités électroniques déterminées, à

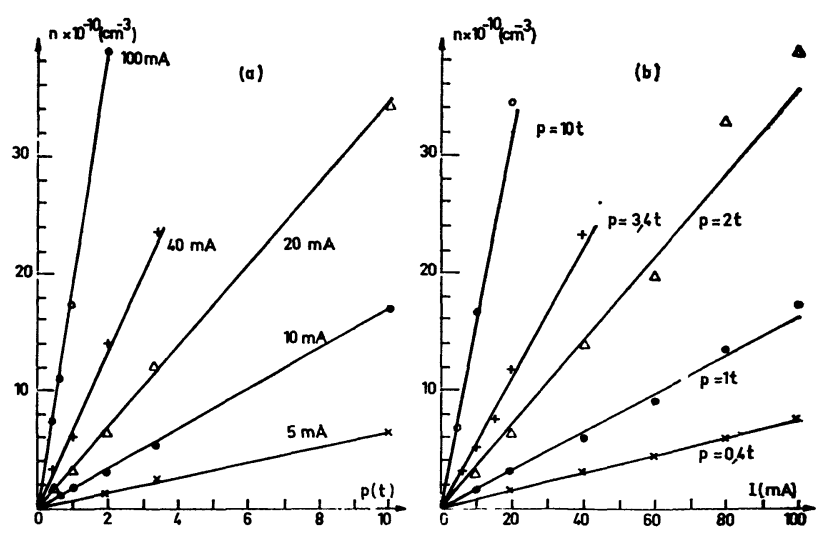

FIG. 1. 
l'aide de la méthode de la cavité, dans un tube de $8 \mathrm{~mm}$ de diamètre $(a)$ en fonction du courant de décharge à pression constante et $(b)$ en fonction de la pression à courant de décharge constant. Nous pouvons constater sur cette figure que les variations de $n_{\mathrm{e}}$ sont linéaires en fonction du courant de décharge (Fig. 1a) et en fonction de la pression (Fig. 1b); nous pouvons donc admettre [voir aussi (4)] que, dans ces conditions, la densité électronique $n_{\mathrm{e}}$ peut s'exprimer par la relation : $n_{\mathrm{e}}=C I p$ [3] où $C$, coefficient de proportionnalité qui dépend du diamètre du tube et de la nature du gaz de la décharge, vaut :

$C=1,7 \times 10^{9} \mathrm{~cm}^{-3} \mathrm{~mA}^{-1}$ torr $^{-1} \quad$ [Néon, $\varnothing 8 \mathrm{~mm}$ ].

Dans un tube de diamètre $4,2 \mathrm{~mm}$ et dans les mêmes domaines de pression et de courant de décharge, l'un des auteurs [5] a montré que la linéarité en fonction de la pression n'était plus vérifiée ; ce résultat confirme celui trouvé par D. Piganeau [6] pour d'autres gaz, dans un tube de diamètre voisin.

2. DensitéS Des atomes métastables. - Les concentrations des atomes métastables $N \mathrm{e}^{*}\left({ }^{3} \mathrm{P}_{0,2}\right)$ et pseudométastables $N e^{*}\left({ }^{3} \mathbf{P}_{1}-{ }^{1} \mathbf{P}_{1}\right)$ présents dans la décharge luminescente du Néon pur ont été déterminés par la méthode de l'absorption optique [3] en fonction des paramètres $I$ et $p$ de la décharge. Rappelons ici, qu'il est important d'effectuer ces mesures de densités de métastables dans un bâti aussi propre que possible [7] de façon à renđre négligeable la destruction des métastables par les impuretés (dégazage des parois...). Les résultats expérimentaux obtenus sont présentés sous la forme d'abaques $\mathrm{Ne}^{*}=f(p)$ pour diverses valeurs du courant de décharge : sur la figure $2 a$ pour le niveau

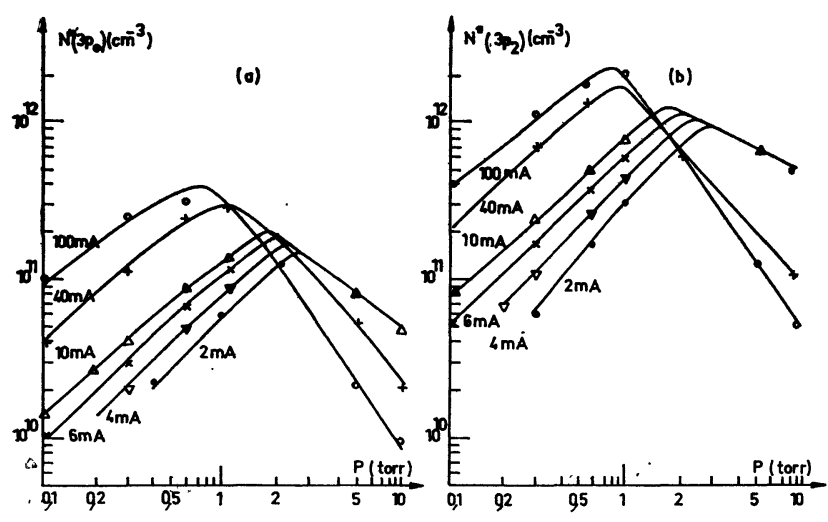

FIG. 2.

${ }^{3} \mathbf{P}_{2}$ et sur la figure $2 b$ pour le niveau ${ }^{3} P_{0}$. Des résultats analogues ont été obtenus en ce qui concerne les niveaux ${ }^{3} \mathbf{P}_{1}$ et ${ }^{1} \mathbf{P}_{1}$ [5]. Il est facile de constater sur les figures $2 a$ et $2 b$ que les courbes $\mathrm{Ne}^{*}=f(p)$ passent par un maximum pour une pression donnée $p_{m}$ et que cette pression diminue quand le courant de décharge augmente. Cette évolution est prévisible à l'aide d'une théorie qui tient compte des processus de formation et de destruction des métastables par collisions ato- miques et électroniques. En effet, en régime stationnaire, la concentration des atomes métastables est donnée par la relation :

$$
N \mathrm{e}^{*}=\frac{N_{0} n_{\mathrm{e}}<\sigma^{M} v>}{\frac{D_{M}}{p \Lambda^{2}}+N_{0}<\sigma_{0}>\bar{v}+n_{\mathrm{e}}<\sigma_{r x} v>}
$$

où $N_{0}$ et $n_{\mathrm{e}}$ sont les concentrations d'atomes neutres et d'électrons respectivement, $\left\langle\sigma^{M} v\right\rangle$ le coefficient d'excitation électronique des métastables, $D_{M}$ est le coefficient de diffusion des métastables, $\Lambda$ est la longueur caractéristique de diffusion, $\left\langle\sigma_{0}\right\rangle$ la section efficace de collision atomique neutre-métastable et $\left\langle\sigma_{r x} v\right\rangle$ le taux de destruction des métastables par collisions électroniques.

La relation (4) peut s'écrire, compte tenu de la relation (3) donnant $n_{\mathrm{e}}$ en fonction du courant et de la pression, sous la forme :

$$
N \mathrm{e}^{*}=\frac{K_{1} I}{\frac{K_{2}}{p}+K_{3} p+K_{4} I p},
$$

où les coefficients $K_{1}, K_{2}, K_{3}, K_{4}$ sont des constantes que nous pouvons relier aux termes de création [numérateur de la relation (4)] et de destruction (dénominateur) des métastables. Si nous dérivons la relation (5) par rapport à $p$ (à $I$ constant) et que nous annulons cette dérivée, nous obtenons pour la pression $p_{m}$ :

$$
p_{m}=\left(\frac{K_{2}}{K_{3}+K_{4} I}\right)^{1 / 2} \text {. }
$$

Les coefficients $K_{2}$ et $K_{3}$, pour les divers niveaux métastables peuvent être calculés à partir de données publiées dans la littérature [8]; on trouve, pour les niveaux ${ }^{3} \mathbf{P}_{0}$ et ${ }^{3} \mathbf{P}_{2}$ :

$$
\begin{array}{ll}
\text { (s }^{-1} \text { Torr) } & K_{2}^{0}=5,4 \times 10^{3} ; K_{2}^{2}=5,4 \times 10^{3} ; \\
\text { (s. Torr) }^{-1} & K_{3}^{0}=3,6 \times 10^{2} ; \quad K_{3}^{2}=1,2 \times 10^{2} .
\end{array}
$$

La détermination expérimentale de $p_{m}$ (à $I$ donné) permet alors d'évaluer le coefficient $K_{4}$ et, par conséquent, le taux de destruction des métastables $\left\langle\sigma_{r x} v\right\rangle$ par collisions électroniques puisque :

$$
K_{4} I p=n_{\mathrm{e}}\left\langle\sigma_{r x} v\right\rangle .
$$

Remarquons que, si $K_{4} I p \gg K_{3} p+K_{2} / p$, la densité des métastables doit tendre vers $K_{1} / K_{4} p$ [cf. relation (5)], c'est-à-dire doit être indépendante du courant $I$; c'est bien ce que nous observons sur les figures $2 a$ et $2 b$ dans le domaine des fortes pressions tant que $I \leqslant 10 \mathrm{~mA}$; par contre, pour $I>10 \mathrm{~mA}$, toujours dans le domaine des fortes pressions, la décroissance de $N e^{*}$ dépend du courant de décharge, ce qui montre que les coefficients $K_{1}$ et $K_{4}$ ne peuvent plus être considérés comme constants ; la variation de ces coefficients est étudiée par l'un des auteurs [9]. En conséquence, nous avons déterminé les coefficients $K_{4}$ dans 
le domaine des courants $I \leqslant 10 \mathrm{~mA}$ et nous avons trouvé :

$$
\left.K_{4}^{0}=K_{4}^{2}=(2,4 \pm 0,1) \times 10^{2} \quad \text { (s.mA.torr }\right)^{-1} .
$$

En ce qui concerne les niveaux pseudo-métastables ${ }^{3} \mathrm{P}_{1}$ et ${ }^{1} \mathrm{P}_{1}$, la pression $p_{m}$ reste sensiblement constante dans le même domaine de courant, ce qui entraîne que les coefficients $K_{4}$ relatifs à ces niveaux sont négligeables.

Des relations (3) et (7), il est possible de tirer la valeur du taux de destruction des métastables ${ }^{3} \mathbf{P}_{0}$ et ${ }^{3} \mathrm{P}_{2}$ :

$$
\begin{aligned}
&\left\langle\sigma_{r x} v>_{{ }^{\mathrm{P}_{0}}}=\left\langle\sigma_{r x} v\right.\right.>_{{ }_{3 \mathrm{P}_{2}}}= \\
&=\frac{K_{4}}{C}=1,4 \times 10^{-7} \mathrm{~cm}^{3} \mathrm{~s}^{-1},
\end{aligned}
$$

valeur voisine de celle calculée par Delpech [4].
Conclusion. - Par la méthode de la cavité (résonnant sur le mode TE 011) et grâce aux techniques de mesures employées (enregistrement continu, contrôle de la température des parois, ...), nous avons pu déterminer avec précision (au moins, en valeurs relatives) la densité électronique dans la colonne positive $(8 \mathrm{~mm}$ de diamètre) d'une décharge luminescente de Néon et nous avons pu vérifier que cette densité $n_{\mathrm{e}}$ était proportionnelle au courant et à la pression de la décharge (Fig. $1 a$ et $1 b$ ). Les déterminations simultanées de $n_{\mathrm{e}}$ et de la concentration des atomes métastables en fonction de la pression et du courant de la décharge nous ont permis d'évaluer le taux de destruction des atomes métastables $\left({ }^{3} \mathrm{P}_{0},{ }^{3} \mathrm{P}_{2}\right)$ du Néon par collisions électroniques. Des études sont en cours pour étendre ces mesures à des décharges de diamètre inférieur $(4,2 \mathrm{~mm})$ à l'aide d'une cavité résonnant sur un mode différent (TM 020).

\section{Bibliographie}

[1] Papoular (R.), Balazard (J.), Application des ondes hyperfréquences et infrarouges à l'étude des plasmas, Dunod, 1965.

[2] Agdur (B.), Enander (B.), J. Appl. Phys., 1962, 33, 2575.

[3] Ricard (A.), J. Physique, 1969, 30, 556.

[4] DelPECH (J. F.), Thèse d'état, Orsay, 1968.
[5] Muzart (J.), Thèse de $3^{e}$ cycle, Toulouse 1970.

[6] Piganeau (D.), Thèse de $3^{\mathrm{e}}$ cycle, Bordeaux, 1968.

[7] Ricard (A.), Rocard (J.-M.), LAUTIER-Gomes (A. M.), C. R. Acad. Sci., 1968, 267, 511.

[8] Phelps (A. V.), Phys. Rev., 1959, 114, 4-1011.

[9] RICARD (A.), Thèse d'état (à paraître). 